\title{
The influence of smoke on $\mathrm{THz}$ imaging
}

\author{
Paweł S. Jung, ${ }^{* 1}$ Mirosław A. Karpierz ${ }^{1}$, Norbert Pałka ${ }^{2}$ and Elzbieta Rurka ${ }^{2}$ \\ ${ }^{1}$ Faculty of Physics, Warsaw University of Technology, Koszykowa 75, 00-662 Warszawa, \\ ${ }^{2}$ Institute of Optoelectronics, Military University of Technology, S. Kaliskiego 2, 00-908 Warszawa
}

Received August 27, 2012; accepted September 23, 2012; published September 30, 2012

\begin{abstract}
The influence of smoke on THz imaging was examined. We tested experimentally a passive THz camera - TS4 from ThruVision Systems Ltd. The camera detects natural human radiation at a frequency of $0.25 \mathrm{THz}$. The distance camera-target could be 3-15 meters Moreover, Time Domain Spectroscopy was used to investigate the influence of the amount of smoke on transmission in the $(0.1 \div 2.5) \mathrm{THz}$ range.
\end{abstract}

Electromagnetic radiation from different ranges is widely applied in military and civilian security to prevent and detect threats. Optoelectronic systems are commonly used not only by the army but also by the police or the fire service. Optical detectors of smoke and fire [1] utilize radiation from ultraviolet (UV), visible (VIS) and infrared (IR) ranges. The above mentioned detectors are usually connected into one fast automatic system for fire detection and extinguishing. These systems are commonly used in server rooms. Detectors based on optical scattering and absorption are applied to identify unknown gases and materials. For example, multiwavelength lidars are used to identify air pollution. X-rays, UV radiation and IR cameras are applied to find the cause of ignition during investigations. A specialized search and rescue group successfully applies cameras with speculum (VIS), IR cameras or seismic and acoustic devices (like geophones) to find people in a fire environment and after building disasters.

Currently, scientists and institutions from around the world are working on terahertz $(\mathrm{THz})$ radiation in order to apply it in the security area. The development of terahertz technology is connected with unique properties of terahertz radiation. The terahertz region of electromagnetic spectrum is defined as $(0.1 \div 10) \mathrm{THz}$ or $(3000 \div 30) \mu \mathrm{m}$ [2] and is located between the domains of infrared and microwaves. These waves are long enough not to be absorbed by nonpolar, nonmetallic materials but still short enough to form an image using optical components [3]. The second advantage of the $\mathrm{THz}$ radiation is that it poses a minimal risk for human beings because of the fact that photon energy is very low $(1 \mathrm{THz}$ radiation coresponds to $4.4 \mathrm{meV}$ ). Another advantage is that the radiation could be used to detect dangerous

\footnotetext{
*E-mail: jung@if.pw.edu.pl
}

materials. Because of the fact that the emissivity of many explosive materials such as Hexogen (RDX), Penthryte (PETN), Octogen (HMX) has some characteristic transmission features in the $\mathrm{THz}$ region $[4,5]$.

a

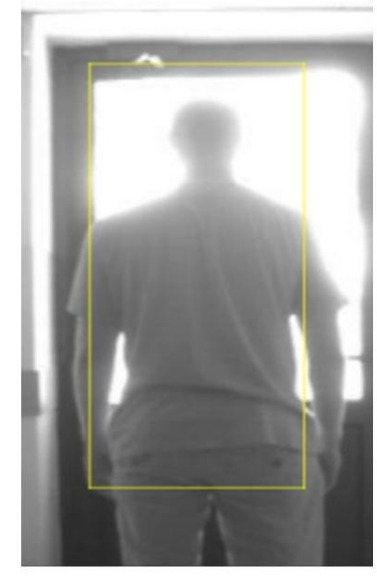

C

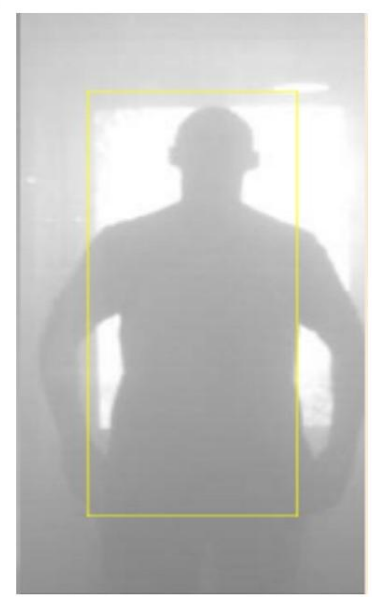

Fig. 1. Pictures in visible range (CCD camera) a) before c) after smokiness and in THz range (TS4) b) before d) after smokiness.

In our work, a screening camera TS4 from ThruVision Systems Ltd. [6] operating in the $\mathrm{THz}$ range was tested in a smoky environment. The number of pixels in the $\mathrm{THz}$ image is $80 \times 50$. The working distance of the camera is 3 15 meters. The camera operating frequency is $0.25 \mathrm{THz}$, 
thanks to the fact that the camera could be used to detect natural human radiation. Clothing transmits well in this range and it is also possible to observe dangerous objects hidden under clothing, such as guns or knives.

In our investigations, a TS4 camera connected with a small visible camera (CCD) was used to observe the scene. They were connected to a computer and software. The images can be recorded independently or a $\mathrm{THz}$ image can be superimposed on a visible image (Fig.1). Smoke aerosol particles were produced from paraffin in a smoke generator. It is a popular method during exercises of the fire service. The aerosol has similar properties like real smoke: the visibility in visible light deteriorates, particle sizes are similar and it is safe for human beings. The distance between the camera and the target (a man) was about 6 meters.

The results of the experiment are seen in Fig. 1 (Figs.1a, b before and Figs. 1c, d after smokiness). The man in the experiment was in a marked position in both cases. Figure 1a presents a visible image of the man. A $\mathrm{THz}$ image superimposed on a visible image is shown in Fig. $1 \mathrm{~b}$ in the yellow frame. The image in the $\mathrm{THz}$ range has a lower quality than from the one from the visible camera, but the contour of the man is still seen. The reason of this is that performance of the passive $\mathrm{THz}$ camera is still limited due to insufficient resolution and sensitivity in comparison to the visible camera. In Figs. 1c and $d$ one can observe the influence of a certain amount of smoke on images in both considered wavelength ranges. In the visible range (Fig. 1c), the image has a lower contrast, but still the man is visible. The man is not seen in the $\mathrm{THz}$ camera.

In simulated fire conditions, the emission of large amounts of smoke could deteriorate the $\mathrm{THz}$ image due to scattering or absorption. The efficiency of scattering depends on the diameter of particles and used radiation. The diameters of smoke particles are from 0.01 to about $10 \mu \mathrm{m}$, depending on a burnt material. Since the particles are smaller than the operation wavelength of the $\mathrm{THz}$ camera $(0.25 \mathrm{THz} 1.2 \mathrm{~mm})$, we can conclude that scattering does not influence the transmission. Its influence is only in the visible and near IR range. We think that the lack of visibility in the $\mathrm{THz}$ range is connected with absorption. In fire (smoke) conditions, $\mathrm{THz}$ radiation is absorbed mostly by water vapour and carbon dioxide $\left(\mathrm{CO}_{2}\right)$ and less by other gases like hydrogen cyanide $(\mathrm{HCN})$, carbon monoxide $(\mathrm{CO})$, hydrochloric $(\mathrm{HCl})$, nitric oxide $(\mathrm{NO})$, and sulphur dioxide $\left(\mathrm{SO}_{2}\right)$. In the examined case it was not only the pure paraffin but also water vapour.

In order to check the influence of smoke on transmission in the $\mathrm{THz}$ range, we exploited Time Domain Spectroscopy (TDS) $[2,4,5]$. The TDS gives us opportunity to measure transmission in the $(0.1 \div 2.5) \mathrm{THz}$ range. In the experiment setup we applied a spectrometer TPS Spectra 3000 from TeraView [7].

Time Domain Spectrometry setup with pulses generated by an $800 \mathrm{~nm}$ femtosecond Ti:Sapphire laser (a pulse duration less than $100 \mathrm{fs}$, repetition rate of $80 \mathrm{MHz}$, and average power of $300 \mathrm{~mW}$ ) is presented in Fig. 2. The laser beam is split into a pump and a probe and directed through a system of mirrors to an emitter and a detector which is based on low temperature grown GaAs dipole antennas. The pump beam is focused on the biased emitter antenna to generate $\mathrm{THz}$ pulses through a photoconductive phenomenon. Such pulses last about $1 \mathrm{ps}$ (Fig. 3) and have a broadband spectrum, usually in the $0.1-3.0 \mathrm{THz}$ range or more.

The transmitted $\mathrm{THz}$ beam can be detected by means of a detector antenna gated using a laser probe beam and a mechanical delay line. A lock-in amplifier and some software are used to collect and process data. The system is purged with dry air to eliminate water vapor.

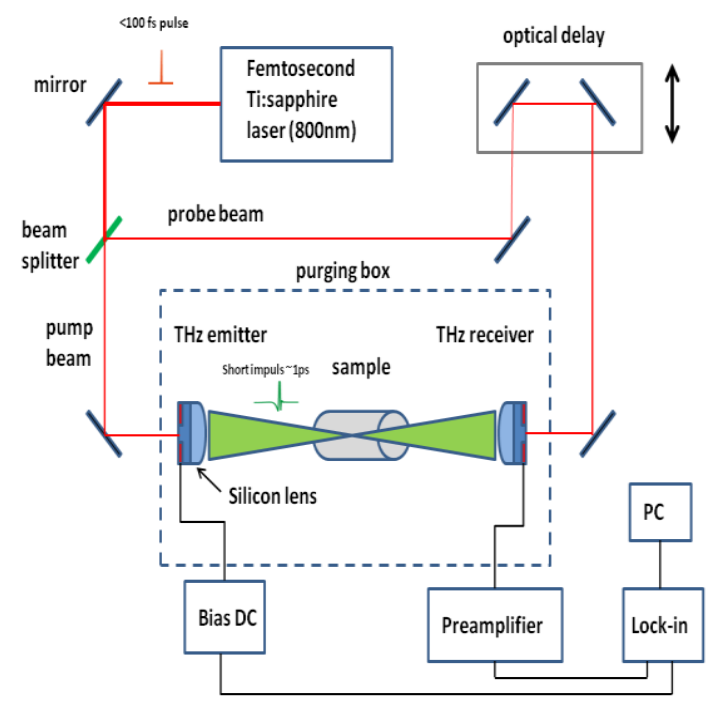

Fig. 2. Setup of Time Domain Spectroscopy.

The analyzed sample had the shape like a cylinder. It was filled with particles of aerosol produced on the basis of paraffin, the same as in the previous experiment. The sample was sealed on both sides with polypropylene foil, transparent to $\mathrm{THz}$ radiation. In all cases, the humidity inside the sample was measured. The sample was placed in the middle between the emitter and the detector, perpendicularly to the THz beam (Fig. 2).

Figure 3 presents impulses measured for the sample as well as the reference impulse acquired for the empty chamber. It is clearly seen from the inset that smoke inside the sample changes the main peak only in a limited way. The main influence of smoke is observed for the position 
of the delay line longer than $39.7 \mathrm{~mm}$. The reference impulse is flat in this region while the smoke inside the sample causes characteristic disturbances.

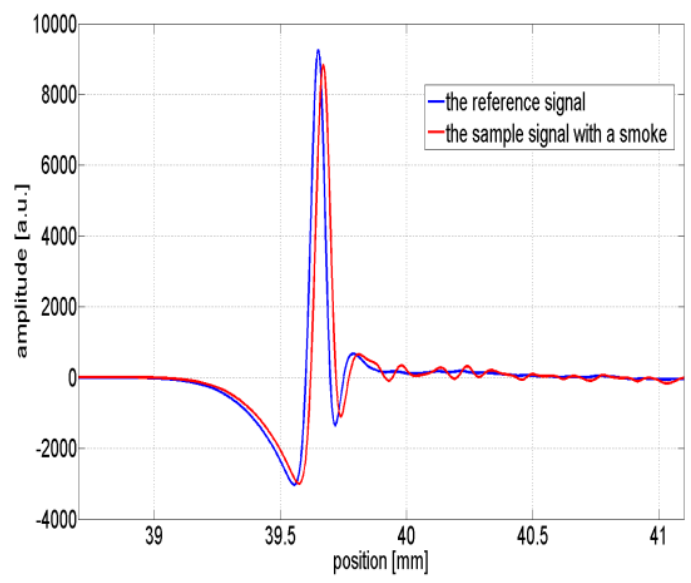

Fig. 3. THz pulses used in TDS: the reference and the sample (parameters of the sample: the volume $196 \mathrm{~cm}^{3}$ and the humidity $52 \%$ ).

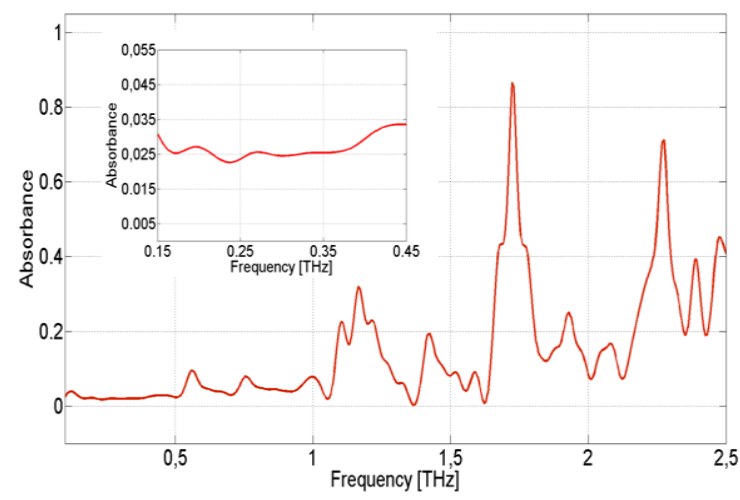

Fig. 4. Measured spectra of absorbance for the sample like above.

The transmission spectra of a sample can be presented as absorbance A:

$$
A=-\log _{10}\left(\frac{T_{M}}{T_{R}}\right)
$$

where $T_{M}$ is the sample spectrum and $T_{R}$ is the reference spectrum.

The absorbance of the considered sample is presented in Fig. 4. Since the sample consisted of water vapour and smoke, the lines connected with water vapour (e.g. at 1.1, $1.7,2.2 \mathrm{THz}$ ) are clearly seen. Obviously, not only the presence of smoke particles but also water vapour has effect on the absorption. The $(0.1 \div 0.5)$ and $(0.5 \div 1) \mathrm{THz}$ ranges compared to higher frequencies with few exceptions have the lowest absorbance. The absorbance for the frequency $0.25 \mathrm{THz}$ for the $10 \mathrm{~cm}$ sample is about 0.025 . While a distance of 6 meters will give the absorbance 60 times higher and it will be 1.5 . It means that the atmosphere with smoke attenuates $10^{-1.5}$ and the level of transmission is about 3\%. It explains the reason of visibility loss in the previous experiment with a $\mathrm{THz}$ passive camera.

Summarizing, in this work the influence of smoke on $\mathrm{THz}$ imaging was examined. Unfortunately, the performance of commercially available passive cameras is still limited due to insufficient resolution and contrast in comparison to other wavelengths, as seen in Fig. 1 a. It has also influence on vision in the case of a cloud of smoke. The $0.25 \mathrm{THz}$ frequency emitted by human beings has for the range of $(0.1 \div 2.5) \mathrm{THz}$ one of the highest transmission (Fig. 4), but absorption is high enough to lose visibility in this range (Fig. 1a). Attenuation of the radiation is related to absorption by aerosol of paraffin and water vapour. The scattering has a small influence on attenuation, because the size of the molecules of the aerosol is smaller than the wavelength of the radiation utilized.

\section{References}

[1] H. Zhao, Advanced Mat. Research 490-495, 927 (2012).

[2] L. Yun-Shik, Principles of Terahertz Science and Technology (Springer 2008).

[3] M. Sypek, M. Makowski, E. Hérault, A. Siemion, A. Siemion, J. Suszek, F. Garet, J.L. Coutaz, Opt. Lett. 37(12), 2214 (2012).

[4] N. Palka, Acta Phys. Pol. A 120(4), 794 (2011).

[5] N. Palka, M. Szustakowski, T. Trzcinski, M. Mozdzonek, Phot. Lett. Poland 3(2),76 (2011).

[6] ThruVision System Ltd., website: www.truvision.com.

[7] Teraview Ltd., website: www.teraview.com 